\title{
Fluctuation of group velocity of Love waves across a dent in the continental crust
}

\author{
Mitsuru Yoshida \\ Earthquake Research Institute, University of Tokyo, 1-1-1 Yayoi, Bunkyo-ku, Tokyo 113-0032, Japan \\ (Received October 27, 1999; Revised May 1, 2000; Accepted May 5, 2000)
}

\begin{abstract}
The dispersion characteristics of group velocity of Love waves are measured across a dent in the continental crust that has a maximum thickness of about $50 \mathrm{~km}$; the numerical modeling analyses are performed using the finite difference method. Measurement of the group velocity over the entire width of the mountain root displays several distinct dispersions. The group velocities for the crustal dent structure are lower than those for the stratified medium with a maximum crustal thickness of the dent at short periods of 20-30 s. The period range becomes longer as the sloping angle of the dent increases or as the crustal thickness of the dent increases. The period indicating a group velocity minimum for the crustal dent structure is shorter than that for the stratified medium with a maximum crustal thickness of the dent. An example of the interpretation of observational data is shown. In the example, the group velocities for the propagation path over the Tibetan Plateau have properties concordant with the above dispersion characteristics.
\end{abstract}

\section{Introduction}

Heterogeneous regions with sloping crustal and upper mantle structures are widely distributed throughout the tectonic regions of the world. In laterally heterogeneous regions with sloping crustal structures, the phase velocity of surface waves depends on the direction of the wave propagation (Boore, 1970 and 1972; Levander, 1985; Yoshida, 1996: paper I). Measurement of the phase velocity over the entire width of the mountain root displays the dispersion characteristics of the root at short periods and the dispersion characteristics of the background structure at longer periods for Rayleigh waves (Levander, 1985) and Love waves (paper I). These characteristics were investigated by numerical modeling analyses. As regards the Eurasian continental paths dominated by lateral variations in the structure, the phase velocity difference between waves travelling in opposite directions has been observed by Levshin et al. (1992).

Using theoretical seismograms, Yamazaki and Ishii (1973) reported that the group velocity of Love waves in a diping structure does not coincide with the group velocity in a stratified medium in the case of a dip angle larger than $3 \mathrm{deg}$. Chun and Yoshii (1977) and Chen and Molnar (1981) analyzed group velocities of surface waves across the Tibetan Plateau with great lateral heterogeneities in its circumference and obtained average properties of the crust and upper mantle. Group velocity maps of East Asia were obtained by Wu and Levshin (1994) for Rayleigh and Love waves and by Wu et al. (1997) for Rayleigh waves. The maps showed that the Tibetan Plateau appears as a prominent low-velocity (about $-15 \%$ from the average) structure in this area, central Tibet appearing as the areas with the lowest velocities (see also Bourjot and Romanowicz, 1992). For paths across the

Copy right $(\mathrm{C}$ The Society of Geomagnetism and Earth, Planetary and Space Sciences (SGEPSS); The Seismological Society of Japan; The Volcanological Society of Japan; The Geodetic Society of Japan; The Japanese Society for Planetary Sciences.
Tibetan Plateau, Tarim basin and the Tien Shan region with large scale crustal dents, extremely low group velocities of Love and Rayleigh waves were observed for periods longer than $20 \mathrm{~s}$ (Levshin and Ritzwoller, 1995). In the above tectonic regions the crustal thickness has regional variations of 40-70 km on the average (Molnar, 1988). In central Eurasia, Levshin et al. (1992) demonstrated a plausible deviation of wave paths near heterogeneous regions with deep crustal dents. However, we cannot adequately interpret group velocity anomalies of surface waves across dented areas of continental crust because we lack precise information with respect to the characteristic of group velocity for sloping structures. At the present stage, we can only pose the most straightforward questions. For example, are the previously described characteristics of phase velocity over the entire width of the mountain root applicable to group velocity? Is there any difference between the group and phase velocity characteristics for paths including a crustal dent? The present paper aims to answer these questions through two-dimensional numerical modeling analyses of Love wave propagation.

\section{Method of Numerical Modeling}

Following Levander (1985, figure 12) and paper I (figure 6(d)), we assume a continental crustal model in which the crust protrudes into the mantle and the surface is flat (Type B). The width $(d)$ and maximum depth (H2) of the dent are assumed to be $250 \mathrm{~km}$ and about $50 \mathrm{~km}$, respectively. The background medium has a crustal thickness $(H 1)$ of $35 \mathrm{~km}$. This model is similar to the configuration (Fig. 1: $\mathrm{X}, \mathrm{Y}, \mathrm{Z}$ ) of the crustal dent in central Europe, which was traced based on figures rendered by Meissner (1986, figure 6.16). Another configuration (Fig. 1: A, B, C) posits the upward-dented crust (Type A). Profiles have been drawn for regions with large variations in the crustal thickness; these serve merely to estimate the sloping angle ( $\theta$ in Fig. 1 ) of the crustal dent. Therefore, numerical modeling is not per- 

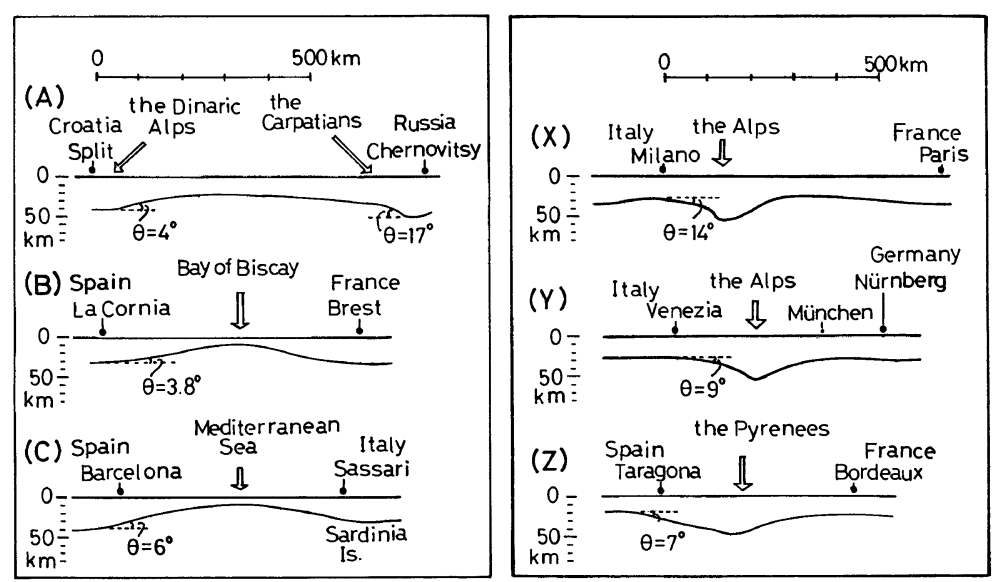

Fig. 1. An example of crustal dents located in central Europe. The sloping angle $(\theta)$ of the Moho is given in degrees. The Moho of the profiles (A, B, C) protrudes onto shallow parts of the crust, whereas that of the profiles $(\mathrm{X}, \mathrm{Y}, \mathrm{Z})$ protrudes into the mantle. The region or city is denoted at the edges of the profiles, along with the mountain range, the sea, or the bay along the profile lines.

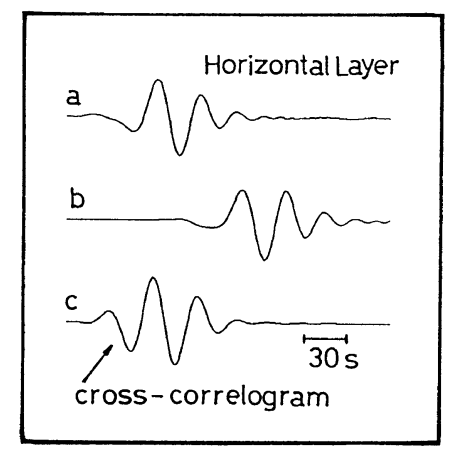

(a)

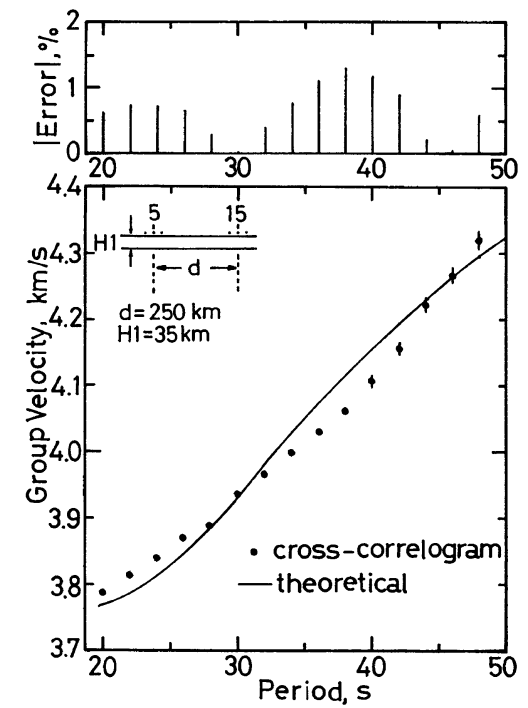

(b)

Fig. 2. (a) An example of the cross-correlogram [c] for synthetic seismograms $[\mathrm{a}, \mathrm{b}]$ recorded at site numbers 5 and 15 , respectively, given in the inset in (b). (b) Average group velocities (solid circles) of the cross-correlograms obtained using three pairs of seismograms recorded at sites 5 and 15 and in their vicinities (dots); a distance of $250 \mathrm{~km}$ is maintained between the two sites. The distance between each dot is 25 $\mathrm{km}$. The seismograms are calculated for a stratified medium with a crustal thickness of $H 1=35 \mathrm{~km}$. The standard deviations are shown by vertical bars. Theoretical group velocities, calculated using normal mode theory, are shown by a solid line. The absolute errors are shown in percentage. formed in each individual profile given in the figure. The sloping angles range between $7 \mathrm{deg}$. and $14 \mathrm{deg}$. for profiles $\mathrm{X}, \mathrm{Y}$, and $\mathrm{Z}$ and between $4 \mathrm{deg}$. and $17 \mathrm{deg}$. for profiles $\mathrm{A}$, $\mathrm{B}$, and $\mathrm{C}$. In all of these profiles, the sloping angle for Type $\mathrm{B}$ seems to be larger than for Type A. Lateral heterogeneity of the crustal dent increases as the sloping angle increases. With an increase in the sloping angle, incident waves scatter under stronger amplification (e.g., Malishewsky, 1987; Momoi, 1987; Its, 1989; paper I). Thus, group velocities for a few select sharply sloping angles for the two configurations are determined in order to determine the characteristic differences between them. Interstation group velocity over the dent is determined by cross-correlation and multiple filtering analysis (MFA) (Dziewonski and Hales, 1972; Landisman et al., 1969). The cross-correlogram $f_{21}(\tau)$ of two seismograms $f_{1}(\tau)$ and $f_{2}(\tau)$ is defined as follows:

$$
f_{21}(\tau)=\int_{-\infty}^{\infty} f_{1}(t+\tau) f_{2}(t) d t
$$

where $f_{1}(t)$ and $f_{2}(t)$ are the signals recorded at the edges of the dent at wave's respective approach and retreat. The function $f_{21}(\tau)$ is equivalent to a signal that would be observed at the second station for a zero phase shift, $\delta$-impulse source situated at the first station. The group velocity can then be calculated by MFA for the cross-correlogram. In the process of MFA, the following filter function is used:

$$
H\left(\omega, \omega_{n}\right)=\exp \left\{-\alpha\left[\left(\omega-\omega_{n}\right) / \omega_{n}\right]^{2}\right\} .
$$

$\omega_{n}$ represents the $n$th center angular frequency. The parameter of $\alpha=25.7$ in Eq. (2) is adequate for a period range of 10-100 s (Yoshida, 1982) and was therefore adopted for use in the present analysis. $S$-wave velocity $(\beta)$ and density $(\rho)$ in the crust are assumed to be $3.85 \mathrm{~km} / \mathrm{s}$ and $3.0 \mathrm{Mg} / \mathrm{m}^{3}$, and $4.75 \mathrm{~km} / \mathrm{s}$ and $3.65 \mathrm{Mg} / \mathrm{m}^{3}$ in the mantle. These elastic parameters are used in Levander (1985) and paper I.

Incident Love waves were calculated using normal mode theory (Saito, 1988) and synthetic seismograms were calculated by the use of the finite difference method (Boore, 1970 and 1972). The finite difference scheme (paper I) performed 


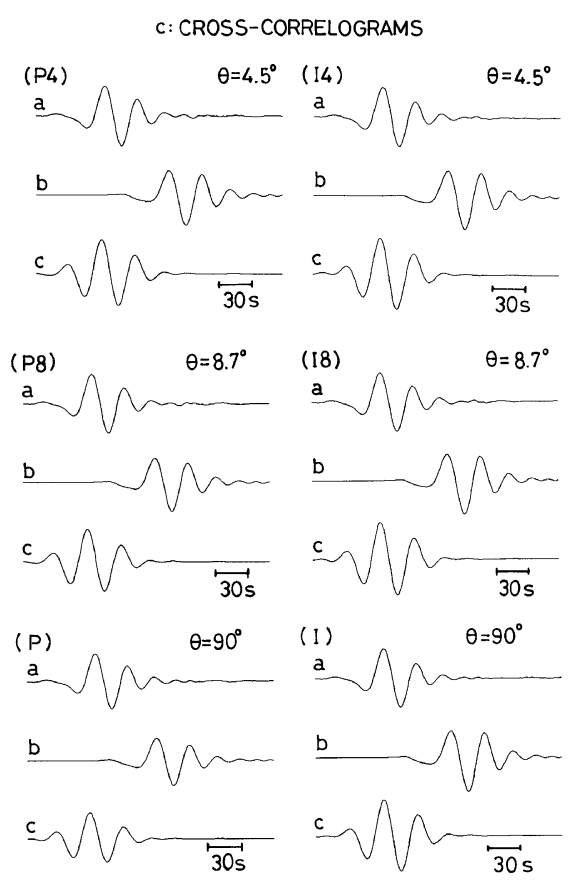

(a)

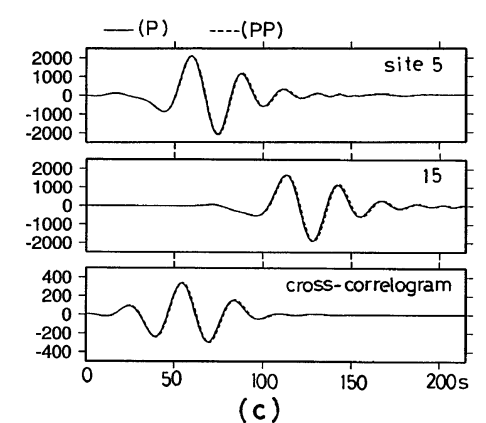

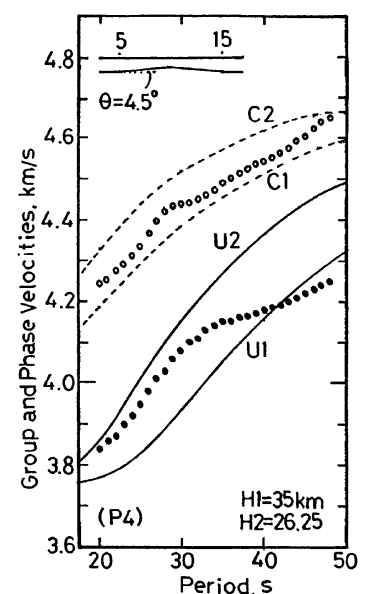
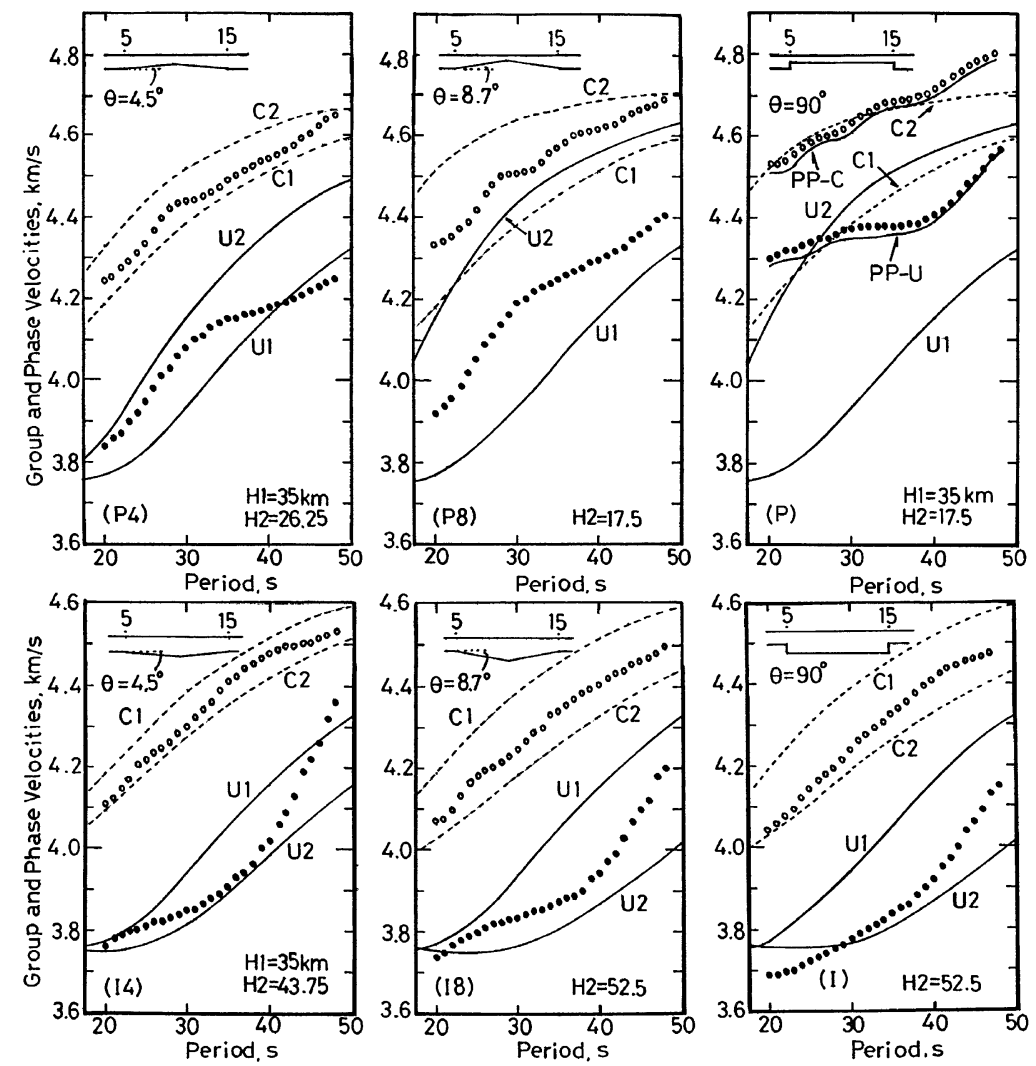

(b)
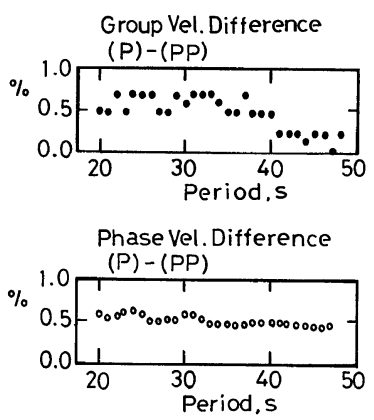

(d)

Fig. 3. (a) Synthetic seismograms [a, b] and their cross-correlograms [c] for Models (P4, P8, P) and (I4, I8, I). The seismograms [a, b] correspond to those at wave's incoming (site 5) and outgoing (site 15) edges. The crustal models are schematically inserted into the figure (b). The Moho of Models (P4, $\mathrm{P} 8, \mathrm{P})$ protrudes onto the shallow parts of the crust, whereas that of Models (I4, I8, I) protrudes into the mantle. The sloping angle $(\theta)$ of the Moho is indicated in the upper right corner of the figure for each model. (b) Group (solid circles) and phase (open circles) velocities for Models (P4, P8, P) and $(\mathrm{I} 4, \mathrm{I} 8, \mathrm{I})$. The crustal thickness $(H 1=35 \mathrm{~km})$ of the background medium is fixed and that $(H 2)$ of the shallowest or deepest point of the dent is indicated in the lower right corner of each figure. Model names are denoted in parentheses. Group and phase velocities for the background medium are denoted by $\mathrm{U} 1$ and $\mathrm{C} 1$, respectively. Group and phase velocities for the stratified medium with a crustal thickness of $H 2$ are denoted by U2 and C2, respectively. PP-C and PP-U are phase and group velocities for Model PP, respectively. For Model PP see the caption in (c). (c) Waveforms for Models P (solid lines) and PP (dotted lines). Model PP consists of the half size of the grid of Model P, vertically. Relative amplitude is indicated in the ordinate and time scale is given in the abscissa in sec. (d) Group (solid circles) and phase (open circles) velocity differences between Models P and PP in percentage.

is as follows: The basic equation for the displacement $(v)$ of Love waves in a Cartesian coordinate system is

$$
\rho v_{t t}=\left[\mu v_{x}\right]_{x}+\left[\mu v_{z}\right]_{z},
$$

where $\mu$ is the rigidity. Subscripts denote partial derivatives. Equation (3) is approximated with the finite difference (Aki and Richards, 1980). The uniform and non-uniform wave guides are located in the left and right sides in the computational space, respectively. The computational space has a horizontal length ( $x$-axis) of $1100 \mathrm{~km}$ and a verti- cal length (z-axis) of $500 \mathrm{~km}$. The total number of grids in the horizontal and vertical directions is 221 and 60, respectively. The boundary condition on the free surface is given by stress free. Initial values are calculated assuming a strike-slip point source and a step function in time as a seismic source. The waves correspond to the fundamental mode Love waves excited at the point with a focal depth of $8.75 \mathrm{~km}$ and a distance of $100 \mathrm{~km}$ in the left-hand direction from the left edge of the computational space. The calculated Love waves are assumed to be incident on the uniform 


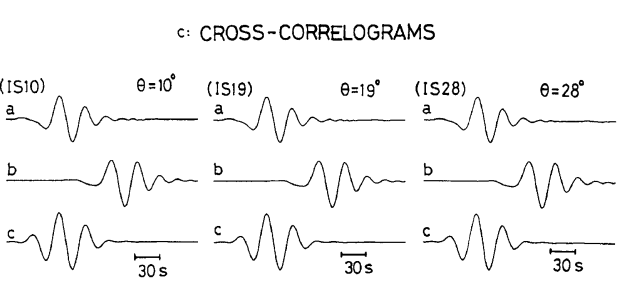

(a)
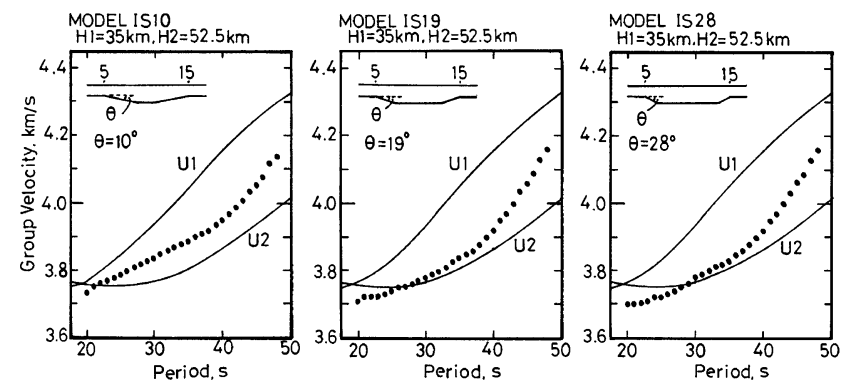

(b)

Fig. 4. (a) Synthetic seismograms [a, b] and their cross-correlograms [c] for Models (IS10, IS19, IS28). The sloping angle $(\theta)$ of the Moho is indicated for each model. (b) Group velocities (solid circles) are determined using the cross-correlograms shown in (a). For U1 and U2, see the caption in Fig. 3(b).

wave guide and propagate towards the non-uniform wave guide with a crustal dent. The elastic parameters are discretized with spatial samples of $\Delta x=5 \mathrm{~km}$ and $\Delta z=8.75$ $\mathrm{km}$ along $x$ - and $z$-axes, respectively. The time sample is $\Delta t$ $=0.7 \mathrm{~s}$. The discretization satisfies the conditions of stability $\left(\Delta t \leq \Delta x \Delta z / \beta \sqrt{\Delta x^{2}+\Delta z^{2}}\right.$ ) and grid dispersion (about 10 grid points per one wavelength) (Aki and Richards, 1980). The A1 absorption condition (Clayton and Engquist, 1977), a zero order paraxial approximation for artificial reflection, was applied at the computational sides and bottom.

The accuracy of the group velocity is shown in Fig. 2, together with an example of synthetic seismograms and their cross-correlogram. First, synthetic seismograms at sites 5 and 15 at a distance $(\mathrm{d})$ of $250 \mathrm{~km}$, for wave incidence from the left, were calculated for the stratified medium $(H 1=$ $35 \mathrm{~km}$ ) and their cross-correlogram was obtained. Second, moving both station sites $25 \mathrm{~km}$ towards the right and left, two cross-correlograms were computed. Next, group velocities were calculated for each cross-correlogram by MFA, as described above. Average group velocities and standard errors are shown in the figure, indicating that the standard errors almost amount to less than $1 \%$. The absolute errors, which also amount to less than $1 \%$, were estimated from the difference between group velocities calculated from Eqs. (1) and (2) and a normal mode solution.

In the following analysis, the crustal dent is located between sites 5 and 15, as given in Fig. 2; the models were schematically inserted into the figure, which shows the group velocity dispersion. A sample of the phase velcity is given as a comparison. The phase velocity is determined by the two-station method (Sato, 1978). In this method, the phase velocity error is estimated to be less than $1 \%$ for periods of 20-50 s (paper I). The dispersion characteristics of the group velocity for dent models are evaluated from the U1 and U2 reference group velocities. U1 is the normal mode solution c: CROSS-CORRELOGRAMS
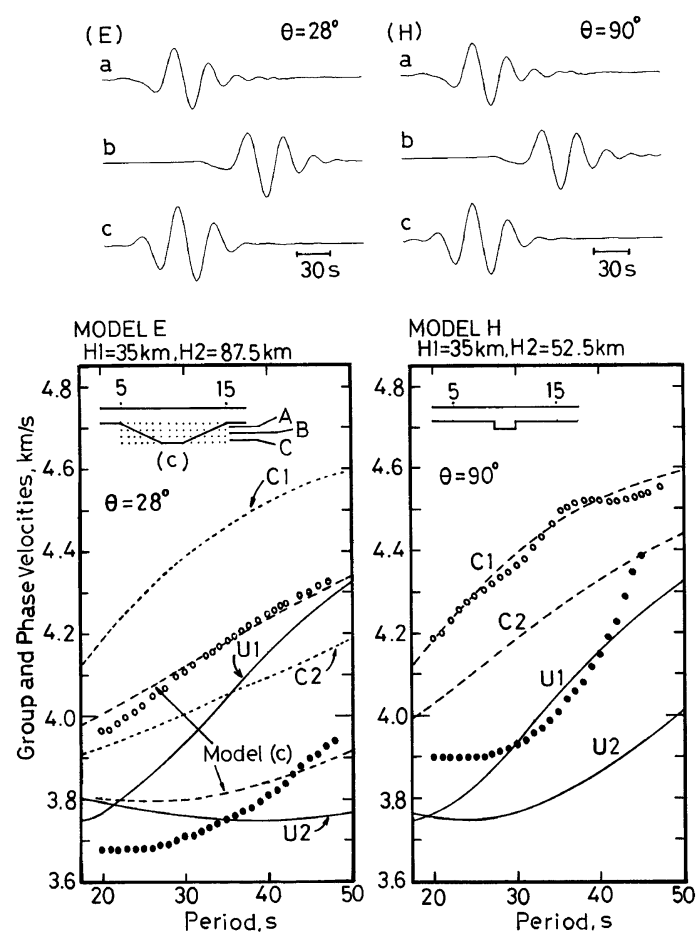

Fig. 5. Top: Synthetic seismograms [a, b] and their cross-correlograms [c] for Models $(E, H)$. The sloping angle $(\theta)$ of the Moho is indicated for each model. Bottom: see legend for Fig. 3(b). Model (c), denoted as (c) in the upper left corner in Model E, is a stratified medium with three horizontal layers $\mathrm{A}, \mathrm{B}$, and $\mathrm{C}$; it replaces for the heterogeneous region with a dent. The elastic parameters for the three layers are synthesized using the weighting coefficients (Yoshida, 1996, equation (1.22)). For (C1, U1) and (C2, U2), see the caption in Fig. 3(b).

for background media with a crustal thickness of $H 1=35$ $\mathrm{km}$, while $\mathrm{U} 2$ is the solution for stratified media with a maximum or minimum crustal thickness of $H 2$, which depends on the models used. The same situation is established for the reference phase velocities of $\mathrm{C} 1$ and $\mathrm{C} 2$.

\section{Result}

In Fig. 3, the cross-correlograms and group velocities for two crustal model configurations are shown together with the phase velocities. Models (P4, P8, P) belong to Type A, while Models (I4, I8, I) to Type B. The differences in the crustal thickness $(H=H 1-H 2$ or $H 2-H 1)$ are $8.75 \mathrm{~km}$ for Models (P4, I4) and $17.5 \mathrm{~km}$ for Models (P8, P, I8, I). We note that the amplitudes of the cross-correlograms (c) for Models (I4, I8, I) are larger than those for Models (P4, P8, $\mathrm{P})$. This suggests that surface waves crossing the crustal dent can be amplified stronger for Type B than for Type A.

For sloping angles of $\theta=4.5 \mathrm{deg}$. and $8.7 \mathrm{deg}$., the phase velocity increases with an increase in period and converges between $\mathrm{C} 1$ and $\mathrm{C} 2$ in Models (P4, P8) and (I4, I8). But at $\theta$ $=4.5 \mathrm{deg}$., the group velocities at periods longer than circa $45 \mathrm{~s}$ are lower than U1 for Model P4 or higher than U1 for Model I4. For $\theta=8.7 \mathrm{deg}$., the group velocities are lower than U2 when approaching a period of $20 \mathrm{~s}$ in the case of Model I8, although they converge between U1 and U2 in the case of Model P8. For the highest sloping angle of $\theta=90$ 
deg., the phase velocity exceeds $\mathrm{C} 2$ at periods longer than 40 $\mathrm{s}$ and the group velocity also exceeds U2 at periods shorter than about $27 \mathrm{~s}$ in the case of Model P. For Model I, which has the same sloping angle as Model P, the phase velocity increases monotonously with an increase in period between $\mathrm{C} 1$ and $\mathrm{C} 2$. The group velocity, however, falls below U2 at periods shorter $30 \mathrm{~s}$. In general, Fig. 3 shows that small fluctuations of phase velocity are stronger for Type A than for Type B. Accordingly, the group velocity dispersion is more unstable for Type A than it is for Type B. This conclusion can be also inferred from the dispersion relationship, namely, that the group velocity depends on the gradient of the phase velocity, as is discussed below. The main goal of the present study is to clarify the dispersion properties for Type B. Thus, the modeling analysis for Type A is limitted to three Models (P4, P8, P). Similar Models will be necessary for future study to gain an understanding of this phenomenon.

The thinnest part of the crust (ex. Model P) has only two grid cells small number of grids. In order to demonstrate the accuracy of original computation with use of such a small grid number for the crust, the waveforms (Fig. 3(c)) for the original (Model P) and half (Model PP) grid size computations were compared. For Model PP the time sample of $0.5 \mathrm{~s}$ was assumed. The waveforms for Models P and PP show a good coincidence. The phase and group velocities for Model PP, shown in Fig. 3(b) along with those for Model $\mathrm{P}$, are slightly lower than those for Model P. The phase and group velocity differences (Fig. 3(d)) between two models are very small, $0.02-0.03 \mathrm{~km}(0.4-0.6 \%)$ and $0.0-0.03 \mathrm{~km}$ $(0.0-0.7 \%)$, respectively.

Figure 4 depicts the group velocities for Models (IS10, IS19, IS28) which have larger sloping angles $(\theta=10,19$, $28 \mathrm{deg}$.); these models posit that the bottom of the mountain root exists at a depth of $H 2=52.5 \mathrm{~km}$. The figure shows that the period range, in which the group velocity becomes lower than the lower reference level of $U 2$, extends towards longer periods as the sloping angle increases. The group velocities for Model IS28 ( $\theta=28 \mathrm{deg}$.) are lower than U2 in a period range from 20 to $30 \mathrm{~s}$, which show a disposition similar to that of Model I $(\theta=90 \mathrm{deg}$.); shown in Fig. 3. The group velocities for Models IS28 and I are 1.3\% and 1.6\% lower than $\mathrm{U} 2$ at a period of $20 \mathrm{~s}$, respectively.

Figure 5 shows the group velocities for three models (E, (c), H). The phase velocity of Model E, which has a maximum crustal thickness of $H 2=87.5 \mathrm{~km}$ and was analyzed in Paper $\mathrm{I}$, increases with an increase in period; this feature is similar to that of Model I. However, the group velocity for Model E is lower than $\mathrm{U} 2$ at periods less than $35 \mathrm{~s}$ and decreases by about $3 \%$ at a period of $20 \mathrm{~s}$. Thus, the group velocity for Model E deviates from U2 more remarkably than that of Model I. The reason for this difference is that the maximum crustal thickness of the dent for Model E is about $35 \mathrm{~km}$ thicker than that of Model I. Model (c), inserted in the figure depicting Model E, is a stratified medium consisting of three horizontal layers and a mantle (paper I), which replaces Model E. Model (c) accounts for the phase velocity dispersion of Model E. However, the group velocities of Model (c), which converge for the most part between U1 and U2, do not agree with those of Model E. Model H, whose crustal dent has a width of $50 \mathrm{~km}$ and is partly included in the middle of the path, is compared with Model I. Phase velocities for Model H are close to $\mathrm{C} 1$, although small oscillations can be detected. However, group velocities for Model $\mathrm{H}$ extensively exceed $\mathrm{U} 1$ for periods of longer than $40 \mathrm{~s}$ and shorter than $30 \mathrm{~s}$. These group velocity anomalies are different from those of Model I; they might be caused by small oscillations in the phase velocity for those periods. Thus the group velocities for Models $\mathrm{H}$ and I feature quite different dispersions, depending on whether the crustal dent is located throughout the whole domain or is partly located along the path.

\section{Discussion}

Here we address the cause of fluctuations in group velocity along the path of a crustal dent. The restriction and availability of group velocity dispersion characteristics described above are also discussed in more detail.

\subsection{Relationship between group and phase velocities}

When the crust inside the dent is shallower or deeper than that of the background medium, the group velocity varies largely as compared with the phase velocity. The phase velocity generally increases monotonously with an increase in period at periods from $20 \mathrm{~s}$ to $50 \mathrm{~s}$. However, the phase velocity curve for sharp crustal dents shows small fluctuations within the period range. The group velocity deviates abruptly near periods of fluctuation of phase velocity. This situation can be described by the following equation:

$$
1 / U=1 / C-T d(1 / C) / d T,
$$

where $T$ refers to the period. Equation (4) implies that the group velocity $(U$ or $1 / U)$ is linearly combined with the gradient of the phase velocity $(C$ or $1 / C$ ) with respect to period. Hence, the group velocity is likely to possess a large overall deviation by small changes in phase velocity. The group velocity dispersions shown in Figs. 3 and 5 suggest that use of group velocities is improper for determining average layered-crustal structures in continental regions with sharp crustal dents. Instead, the use of phase velocities (e.g., Romanowicz, 1982; Brandon and Romanowicz, 1986; Curtis and Woodhouse, 1997) is favorable for the inversion of such laterally heterogeneous regions, especially for regions with a mountain root (Models I, E). In regions, in which the crust inside the dent becomes abruptly shallow (Model P), the phase velocity, as well as the group velocity, is not a feasible measure of inversion because the phase velocity exceeds the upper limit of $\mathrm{C} 2$ at longer periods.

\subsection{Effects of Love wave scattering}

Let us consider here the scattering effects due to the crustal dent. Characteristic features of scattering of Love waves across the dent can be delineated as follows (paper I, figure 40). If we define the propagation from thinner to thicker crusts as "downdip propagation" and the reverse case as "updip propagation", converted $S H$ waves are generated at short and long periods for the downdip and updip propagations, respectively. The reason is that the energy of Love waves at short and long periods decreases for the downdip and updip propagations, respectively. This scattering phenomenon is illustrated in the inset of Fig. 6, which shows the local mode displacement eigenfunction at a period of $30 \mathrm{~s}$. In the inset, it is assumed that incident Love waves propagate from left to right. The inset also shows that a representative depth 


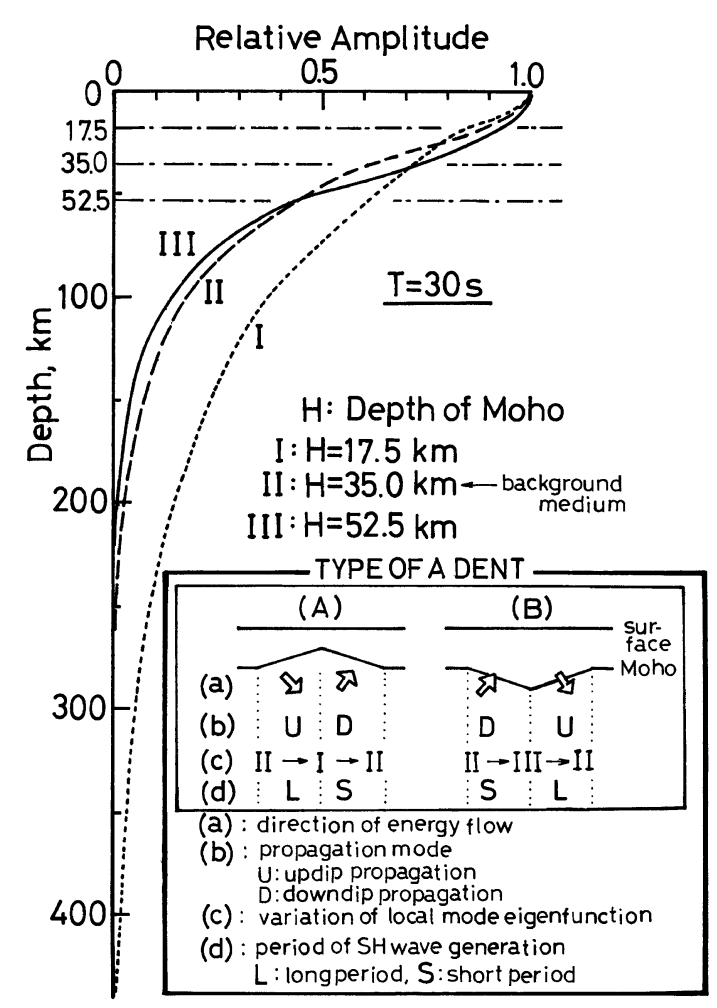

Fig. 6. Normalized displacement eigenfunctions for a period of $30 \mathrm{~s}$. The eigenfunctions of I, II, and III are calculated for stratified media with a crustal thickness of $17.5,35.0$, and $52.5 \mathrm{~km}$, respectively. In the inset, the type of crustal dent is schematically depicted, together with the associated phenomena of the wave propagation. Type A crust inside the dent is thinner than that of the background medium, whereas in the case of Type B, this feature is reversed. The associated phenomena (a, b, c, d) are illustrated based on the assumption that Love waves propagate from left to right. Note that characteristics of the wave propagation vary reversely for Types A and B. See the text for details.

dominated with Love wave energy shifts towards shallower or deeper parts as local mode eigenfunction varies. Here, the representative depth is defined as "the depth corresponding to a quater of one wavelength of Love waves of a period of 30 $\mathrm{s}$, the waves having largest energy in incident waves." Furthermore, Love waves suffer a corresponding interference of short- and long-period body waves in the first and last halves of the dent for Type $B$, because the propagation mode in the first and last halves of the dent for Type B belongs to the downdip and updip propagations, respectively. The sequence is reversed for Type A. Thus, the process of body wave interference is quite different for Types A and B.

The large deviation of group velocities due to the effect of scattering occurs at periods less than $30 \mathrm{~s}$ for both Types A (Model P) and B (Model I). This feature might also be explained by Love wave properties, namely, that the wave energy is trapped in the crust and the waves are influenced by the medium mostly within a quater of one wavelength (Dahlen and Tromp, 1998, pp. 449). For stratified media with a crustral thickness of $H 1=17.5,35$, and $52.5 \mathrm{~km}$, the wavelength at a period of $30 \mathrm{~s}$ is about 139, 130, and $126 \mathrm{~km}$. Hence, the variation in crustal thickness is sensitive to Love waves of periods less than $30 \mathrm{~s}$ for both Types A and $\mathrm{B}$, as far as the crustal dent varies from 20 to $50 \mathrm{~km}$ in

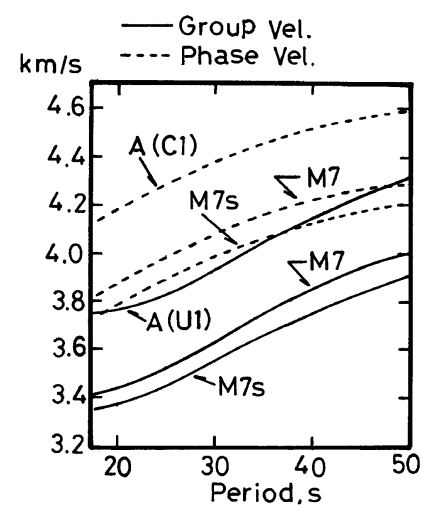

Fig. 7. Group and phase velocities calculated for three Models (A, M7, M7s). Model A, whose group and phase velocities are denoted respectively by $\mathrm{U} 1$ and $\mathrm{C} 1$, has a crustal thickness of $H 1=35 \mathrm{~km}$ and is used as the background medium. The crust and upper mantle models for Europe, designated as M7 (Nolet, 1977) and M7s (Snieder, 1988), have a crustal thickness of $33 \mathrm{~km}$. $S$-wave velocity at the top $170 \mathrm{~km}$ in Model $\mathrm{M} 7 \mathrm{~s}$ is $2 \%$ lower than that of Model M7. Note that the group and phase velocities for the crustal models used in the present analysis are about 0.4 $\mathrm{km} / \mathrm{s}$ higher than those in European models.

depth. It is also noted that for Model P (Type A), both phase and group velocities are unstable at periods longer than 40 s. This peculiarity might be explained by the interference of body waves (paper I); in particular, the body wave generation is stronger according to variations in the crustal thickness at the shallower sections. On the other hand, stable phase and group velocities at periods longer than $40 \mathrm{~s}$ for Model I (Type B) can be explained by weaker scattering patterns as regards variations in the crustal thickness at the deeper sections; this holds even if the variation of crustal thickness $(\Delta H=H 2-H 1=17.5 \mathrm{~km})$ of Model $\mathrm{I}$ is equal to that $(\Delta H=H 1-H 2=17.5 \mathrm{~km})$ of Model P.

\subsection{Properties of background medium group velocity dispersion}

The elastic parameters used for the present analysis are different from those used in Europe (Fig. 1). The group and phase velocity dispersion curves calculated for the crustal and upper mantle models of M7 (Nolet, 1977) and M7s (Snieder, 1988), used in Europe, are shown in Fig. 7 for comparison. Both models have a crustal thickness of $33 \mathrm{~km}$. Model M7s is equal to Model M7, except that the $S$-wave velocity at the top $170 \mathrm{~km}$ is $2 \%$ lower than of Model M7. Snieder (1988) reported that Model M7 represents the Scandinavian shield, which has an anomalously high $S$-wave velocity; Model M7s represents central Europe. We note from the figure that both the group (U1) and phase (C1) velocities for the background medium (designated as " $A$ " in the figure), used in the present analysis, are about $0.4 \mathrm{~km} / \mathrm{s}(9-10 \%)$ higher than those of the models used in Europe. This is because $S$-wave velocities at the top $90 \mathrm{~km}$ for Model M7s are 3.400, 3.714, and 4.341 $\mathrm{km} / \mathrm{s}$ at depths of 0-20, 20-33, and 33-90 km, respectively; these $S$-wave velocities are 12,4 , and $9 \%$ lower than those used in the present analysis.

\subsection{Interpretation of an observed group velocity disper- sion}

Here, we propose an interpretation of the observed group velocities of Love waves which traversed the Tibetan Plateau 

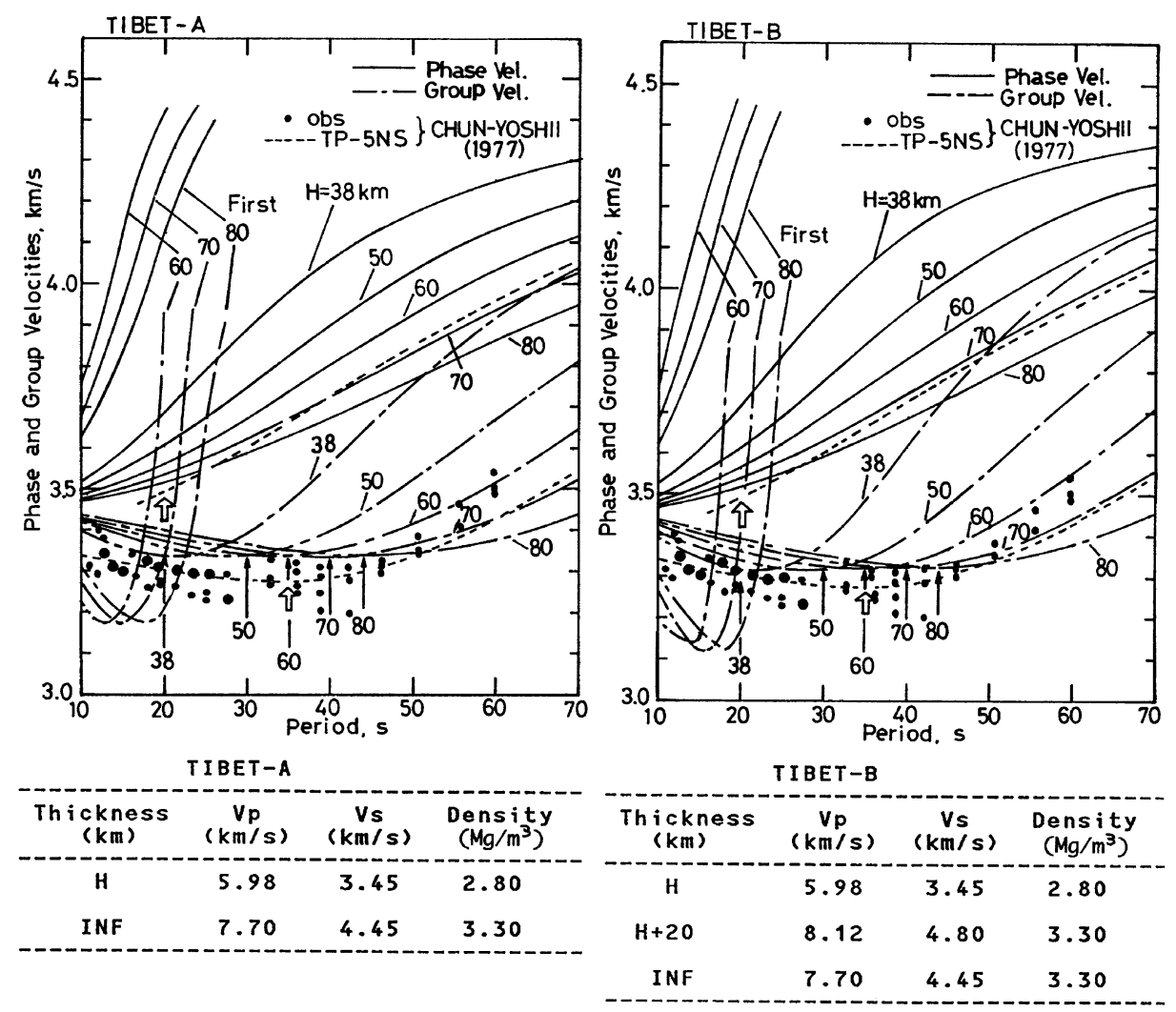

Fig. 8. Observed and calculated group velocities of fundamental mode Love waves across the Tibetan Plateau with a large-scale continental crustal dent. Observed group velocities (obs: dots) are those reported by Chun and Yoshii (1977), in which the smallest dot indicates one data point and the larger dot indicates two or more points superimposed. Group velocities (dot-dash lines), calculated as functions of the crustal thickness $(H=38,50,60,70,80$ $\mathrm{km}$ ) of the dent, are those for the stratified medium models of TIBET-A and TIBET-B, whose elastic parameters are given below. Model TIBET-B has a high $S$-wave velocity of $4.80 \mathrm{~km} / \mathrm{s}$ for the uppermost mantle of a thickness of $20 \mathrm{~km}$, whereas Model TIBET-A does not have a thin high velocity layer. Phase velocities (solid lines) calculated for TIBET-A or TIBET-B are also shown, together with those for Model TP-5NS, which was constructed by Chun and Yoshii (1977) to fit the observed group velocities (obs: dots). Group and phase velocities denoted by "First" are those of the first higher mode calculated for TIBET-A or TIBET-B. Upward-pointing solid arrows indicate the location of a group velocity minimum for a stratified medium with a crustal thickness of $H$. Upward-pointing open arrows at periods of $20 \mathrm{~s}$ and $35 \mathrm{~s}$ indicate locations of minima of observed phase and group velocities in a period range of 20-70 s. The crustal thickness of $H=38 \mathrm{~km}$ is close to that of the Indian Shield (Honda and Sakai, 1988; Tanimoto, 1995), whereas thickness of $H=80 \mathrm{~km}$ corresponds to that of the Tibetan Plateau at an average of 70-80 km (Hirn et al., 1984; Wittlinger et al., 1996; Kind et al., 1996) with a partially molten layer (Nelson et al., 1996; Brown et al., 1996; Makovsky et al., 1996) and a maximum of 85 km (Chen and Molnar, 1981) or $78 \mathrm{~km}$ (Curtis and Woodhouse, 1997).

in terms of the wave propagation over the crustal dent. In Fig. 8, the observed group velocities (obs) of Love waves reported by Chun and Yoshii (1977) are shown along with phase and group velocities for the stratified media (TIBET-A and TIBET-B) calculated as functions of the crustal thickness $(H=38,50,60,70,80 \mathrm{~km})$. The elastic parameters of the $S$-wave velocity of $3.45 \mathrm{~km} / \mathrm{s}$ for the crust and $4.5 \mathrm{~km} / \mathrm{s}$ for the mantle, estimated by Kind et al. (1996) and Chun and Yoshii (1977), respectively, are used. The model TIBETB shows a high- $S$ velocity of $4.80 \mathrm{~km} / \mathrm{s}$ for the uppermost mantle of a thickness of $20 \mathrm{~km}$ (Chen and Molnar, 1981). On the other hand, the model TIBET-A does not have the thin layer. Densities of 2.8 and $3.3 \mathrm{Mg} / \mathrm{m}^{3}$ for the crust and mantle, respectively, are taken from Chun and Yoshii (1977). The figure shows that the dispersion characteristics for TIBET-A and TIBET-B are very similar to each other except that the phase and group velocities for the latter are slightly higher at longer periods than those of the former.

Phase and group velocities calculated for a stratified model of TP-5NS with three crustal layers and a mantle, which was constructed by Chun and Yoshii (1977) for the "pure Tibetan path" with path lengths of about $2000 \mathrm{~km}$ to fit the observed group velocities are also shown in Fig. 8. We temporarily assume the phase velocities calculated for TP-5NS as "observed phase velocities" for the Tibetan Plateau. According to Levander (1985) and paper I, the phase velocity of surface waves over the entire width of the mountain root displays the characteristics of the dispersion of the root near a period of $20 \mathrm{~s}$ and increases monotonously with the increase in period. It also displays the characteristics of background structures at longer periods near $90 \mathrm{~s}$. Similar characteristics can be observed in the figure, which shows that the "observed phase velocities" are close to those for TIBET-A or TIBET-B of a crustal thickness of 70-80 km at a period of $20 \mathrm{~s}$ (upward open arrow); these velocities increase with the increase in period.

As is pointed out in the dispersion characteristics of Models (IS10, IS19, IS28, I, E), the group velocities over the entire width of the crustal dent are lower than those of stratified media with a maximum crustal thickness of the dent at short periods. Furthermore, the period indicating a group velocity minimum for the dent is shorter than that of a strat- 
(c) CROSS-CORRELOGRAMS
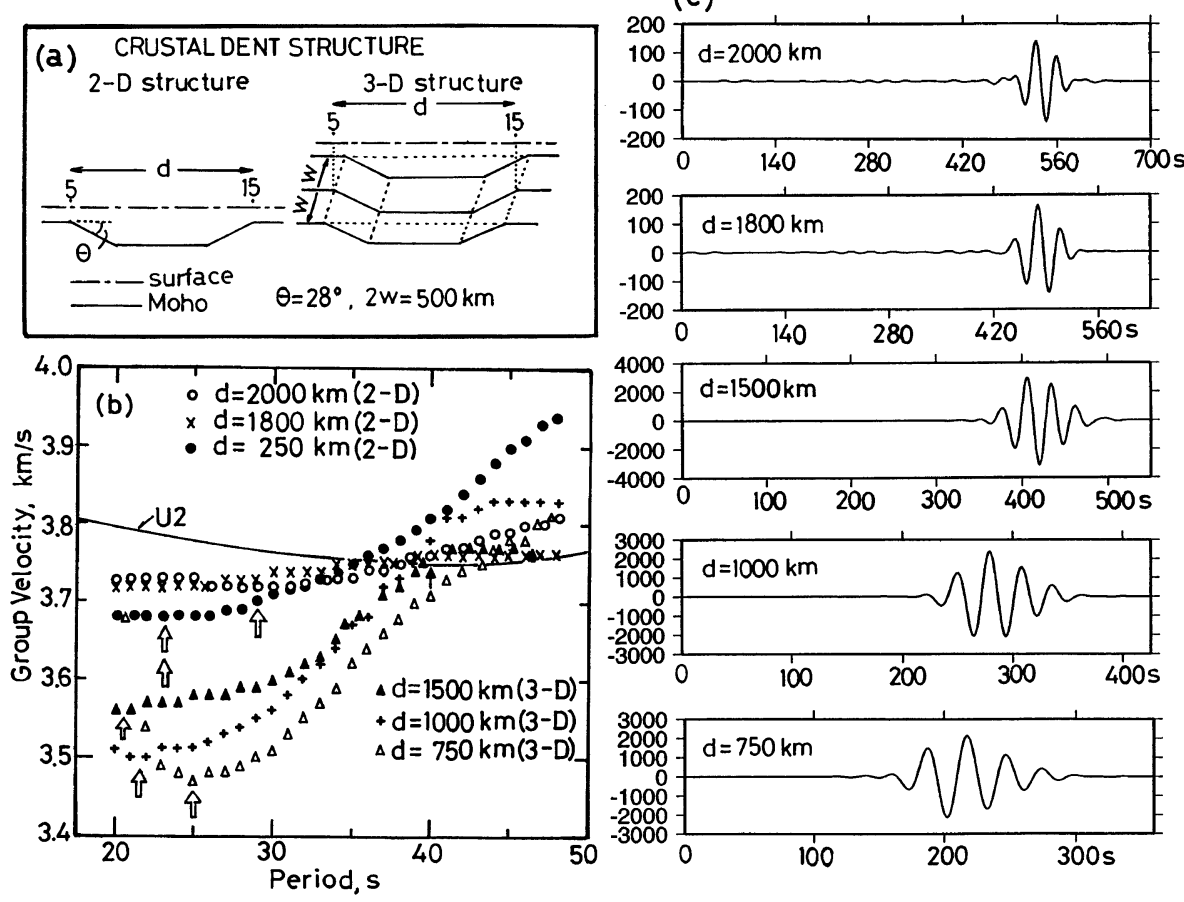

Fig. 9. (a) Schematic view of 2-D and 3-D crustal structures of mountain root models. The path length between sites 5 and 15 along the great circle and the sloping angle of the Moho are denoted by $\mathrm{d}$ and $\theta$, respectively. The width of the crustal dent in the transverse direction is denoted by $2 \mathrm{w}$. Waves are incident from the left for 2-D and 3-D structures, normal to the dent front lying on site 5 in the transverse direction for 3-D structure. (b) Group velocities for 2-D $(d=2000,1800$ and $250 \mathrm{~km})$ and 3-D $(d=1500,1000$ and $750 \mathrm{~km})$ crustral structures, determined using cross-correlograms given in (c). Group velocities for $d=250 \mathrm{~km}$ (Model E) are taken from Fig. 5. U2 is the normal mode solution of group velocity for a stratified medium with a maximum crustal thickness of $H 2(=87.5 \mathrm{~km})$. An upward-pointing solid arrow at a period of $40 \mathrm{~s}$ indicates the location of a group velocity minimum with a crustal thickness of $H 2$. Upward-pointing open arrows at periods of 20-30 s indicate locations of minima of group velocities for 2-D or 3-D structures. (c) Cross-correlograms between sites 5 and 15 for 2-D or 3-D crustal structures with path lengths of d. Relative amplitude is indicated in the ordinate and time scale is given in the abscissa in sec.

ified medium with a maximum crustal thickness of a dent. These characteristics can be also seen in Fig. 8. The observed group velocities for the Tibetan Plateau are less than those for TIBET-A or TIBET-B with a crustal thickness of $H=70-80 \mathrm{~km}$ at periods of $20-50 \mathrm{~s}$. The period indicating a group velocity minimum (upward open arrow) is located at a period of $35 \mathrm{~s}$, which is about 5-10 s shorter than those of a stratified medium with a crustal thickness of $H=70-80 \mathrm{~km}$. Thus, we can deduce that the relationship between the group and phase velocities, shown in Models I (Fig. 3(b)) and E (Fig. 5), is found to exist between observed group and phase velocities for the Tibetan Plateau. These results suggest that the effects of lateral heterogeneities can be reflected upon the observed group velocities. Such heterogeneities include two sloping superimposed crustal structures with a dip angle of about 10 deg., several vertical 20-km steps of the Moho at depths of $50-70 \mathrm{~km}$ in the Indian side of the Tibetan Plateau (Allegre et al., 1984), and the sloping crustal structure in the eastern side of the Plateau. At $H=80 \mathrm{~km}$, the phase and group velocities for TIBET-A and TIBET-B, if calculated for the spherical earth, can be expected to approach "observed velocities" at periods of longer than $50 \mathrm{~s}$. This is because long period surface wave velocities are higher for the spherical earth than for the flat earth (e.g., Schwab and Knopoff, 1972).

\subsection{Applicability of mountain root models and data qual- ity}

The observed dispersion relation (Chun and Yoshii, 1977) was obtained based on waveform data for relatively long travelling paths: the path is about $2000 \mathrm{~km}$. The Tibetan Plateau has a similar horizontal scale. In order to discuss the dispersion relation for the Tibetan Plateau, the mountain root models, which have much larger horizontal scales than those simulated previously, are needed. Therefore, several 2-D and 3-D models (Fig. 9(a)) for longer paths were analyzed. Our attention focussed on whether the group velocity dispersion, as seen in Models I and E with a path length of $d=250 \mathrm{~km}$, can be obtained or not for long paths. The cross-correlograms between sites 5 and 15 were calculated and are shown in Fig. 9(c). Using these cross-correlograms group velocities (Fig. 9(b)) were determined. The main dispersion relations obtained are as follows:

A-1) The group velocities for path lengths of $2000 \mathrm{~km}$ (V2000) and $1800 \mathrm{~km}$ (V1800) are similar to those for $d=250 \mathrm{~km}$ (Model E) for periods considered here as a whole.

A-2) V2000 and V1800 are slightly higher and lower than the group velocities for Model $\mathrm{E}$ at periods shorter and longer than about $35 \mathrm{~s}$, respectively; At long periods, V2000 and V1800 approach U2 (the group velocity for the stratified medium with a maximum crustal thickness 
of $H 2(=87.5 \mathrm{~km})$ for Model E). This might be caused from the reason that the scattering effects within the dent became weaker compared to those for Model E, because the path lengths became much longer.

A-3) V2000 and V1800 are lower than U2 at short periods; The periods indicating a group velocity minimum (upward-pointing open arrows) for the dent structures are shorter than that (an upward-pointing solid arrow) for the stratified medium. 3-D simulations were performed to clarify the effect of obstacles in the transverse direction, following the computational procedure of paper I. The boundary of the crustal dent in the transverse direction is assumed to be located at a distance of $250 \mathrm{~km}$ from the great circle path along sites 5 and 15 . The width $(2 w=500 \mathrm{~km})$ of the crustal dent is the size comparable to the scale of the Tibetan Plateau in the north-south direction. The dispersion relations obtained from 3-D models for path lengths of 1500, 1000, and $750 \mathrm{~km}$ are featured as follows.

A-4) The group velocities at periods of 20-35 s are greatly lowered. This might be caused by the effects of scattering and diffraction in 3-D crustal dent structures; Characteristic A-3) can be clearly observed in 3-D models.

A-5) The dispersion relations for 3-D structures approach those for 2-D structures with an increase in path length. Taking into consideration the results of numerical simulations above, it can be deduced that Characteristic A-3) are detected even for long paths near $2000 \mathrm{~km}$.

Next, we discuss the quality of the data of Chun and Yoshii (1977). As is discussed by them, the data is carefully selected to construct a crustal structure (TP-5NS) for the Tibetan Plateau. The data (solid circles in Fig. 8) is essentially not the average of observed data but is rigorously refined as follows:

B-1) The data of five paths (3-NDI, 5-NDI, 9-NDI, 14-NDI, $15-\mathrm{NDI})$ is selected from all the data of 18 paths for the "pure Tibetan" path; (NDI: the station located in the middle part of the northern India); The boundary around the Tibetan Plateau is located at a distance of 250-600 $\mathrm{km}$ from the great circle paths in the transverse direction.

B-2) The effect of sedimentary layer in the Gangetic Basin near the station is corrected.

B-3) The effect of topography of the Himalayan Range is also corrected.

B-4) The data of the non-Tibetan portion of the paths, such as the paths of structually complex region in the southwest of Tibet is excluded. Chen and Molnar (1981, figure $3 b$ ) determined group velocities of Love waves over the Tibetan Plateau. Among the observed data,

B-5) The dispersion relations for selected paths (III-NDI, IVNDI, VII-NDI, VIII-NDI, IX-NDI), for the "pure Tibetan" path, agree quite well with those of Chun and Yoshii (1977) at periods of 20-60 s; The selected paths traverse almost the central part of the Tibetan Plateau; The distance from the great circle paths to the boundary around the Tibetan Plateau, in the transverse direction, extends to more than $500 \mathrm{~km}$.

Furthermore, group velocity distributions of Love waves for the Tibetan Plateau, obtained by Wu and Levshin (1994), are in harmony with the dispersion relation for TP-5NS at periods of 30-70 s. These data environment firmly suggests that the group velocity data in Fig. 8 are reliable and are in high quality. Thus, the dispersion relationship discussed in Section 4.4 can be supported by both the results of modeling analyses for long paths and high quality data. The 3-D models analyzed above work only for structure with cylindrical symmetry (at most) and no real structure has that symmetry. In heterogeneous 3-D media, anomalies of particle motions (Levshin et al., 1992), interconversions between Love and Rayleigh waves (e.g., Kennett, 1984; Snieder, 1986; Bostock, 1991) and effects of diffraction (e.g., Tanimoto, 1990; Pollitz, 1994; Pedersen et al., 1996) are significant. The coupling between Love and Rayleigh waves in crustal dents increases with an increase in angle of oblique incidence (Pedersen et al., 1998). These 3-D effects make variations of amplitude and phase velocity of Love waves (paper I), depending on the the angle of oblique incidence. Then, the group velocity is bound to fluctuate in accordance with variations of the phase velocity through Eq. (4).

\section{Conclusions}

In this study, numerical modeling analyses were employed to investigate the dispersion characteristics of the group velocity of Love waves across dented continental crust in a period range of 20-50 s. The crustal thickness of the background medium and the crustal dent were assumed to be 35 $\mathrm{km}$ and $52.5-87.5 \mathrm{~km}$, respectively. The crustal dent was assumed to have an entire width of $250-2000 \mathrm{~km}$. The characteristics of the group velocity were investigated by varying the sloping angle of the dent in a range of 4.0-90 deg. Measurement of group velocity over the entire with of the mountain root displayed the following distinct dispersions: 1) at short periods (20-30 s), the group velocities are lower than those of a stratified medium with a maximum crustal thickness of the dent; 2) period range showing low group velocities extends with an increase in the sloping angle or in the maximum crustal thickness of the dent; 3 ) the period indicating a group velocity minimum for the entire width of the dent is shorter than that of a stratified medium with a maximum crustal dent thickness. These fluctuations in group velocity were observed in Love waves across the Tibetan Plateau, which has a large scale continental crustal dent. The fluctuation describes observed group velocities as being lower than those of a stratified medium with a crustal thickness of $70-80 \mathrm{~km}$ at periods of $20-50 \mathrm{~s}$; furthermore, a period of $35 \mathrm{~s}$ indicates an observed group velocity minimum that is shorter by $5-10 \mathrm{~s}$ than those of a stratified medium with a crustal thickness of 70-80 km.

The dispersion characteristics for the upward-dented crust (Type A) show that small fluctuations of phase velocity are stronger than those associated with the mountain root (Type B). Type A group velocity dispersion is thus more unstable than for Type B velocity dispersion, as far as the background medium has a crustal thickness of $35 \mathrm{~km}$.

Group velocities of surface waves which traversed purely the dented continental crust are scarcely observed. Accumulation of observational data for specified regions in Eurasia, as well as refinement of numerical models, using spherical coordinates, would be important for future study to quanti- 
tatively establish a dispersion law for group velocity over a large scale crustal dent.

Acknowledgments. The computations were carried out at the Earthquake Information Center, Earthquake Research Institute, and at the Information Technology Center, University of Tokyo. The author wishes to thank two anonymous reviewers and Prof. Toshiro Tanimoto, University of California, USA, for useful comments.

\section{References}

Aki, K. and P. G. Richards, Quantitative Seismlogy: Theory and Methods, pp. 728-730, W. H. Freeman and Co., San Francisco, 1980.

Allegre, C. J., V. Courtillot, P. Tapponnier, A. Hirn, M. Mattauer, C. Loulon, J. J. Jaeger, J. Achache, U. Scharer, J. Marcoux, J. P. Bourg, J. Girardeau, R. Armijo, C. Gariepy, C. Gopel, L. Jindong, X. Xuchang, C. Chenfa, L. Guangquin, L. Baoyu, T. Jiwen, W. Naiwen, C. Guoming, H. Tonglin, W. Xibin, D. Wanming, S. Huaibin, C. Yougong, Z. Ji, Q. Hongrong, B. Peisheng, W. Bixiang, Z. Yaoxiu, and R. Xu, Structure and evolution of the Himalaya-Tibet orogenic belt, Nature, 307, 17-22, 1984.

Boore, D. M., Love waves in nonuniform wave guides: Finite difference calculations, J. Geophys. Res., 75, 1512-1527, 1970.

Boore, D. M., Finite difference methods for seismic propagation in heterogeneous materials, in Methods in Computational Physics, Volume 11, edited by B. A. Bolt, pp. 1-37, Academic Press, 1972.

Bostock, M. G., Surface wave scattering from 3-D obstacles, Geophys. J. Int., 104, 351-370, 1991.

Bourjot, L. and B. Romanowicz, Crust and upper mantle tomography in Tibet using surface waves, Geophys. Res. Lett., 19, 881-884, 1992.

Brandon, C. and B. Romanowicz, A "non-lid" zone in the central Chan-thang platform of Tibet: Evidence from pure path phase velocity measurements of long period Rayleigh waves, J. Geophys. Res., 91, 6547-6564, 1986.

Brown, L. D., W. Zhao, K. D. Nelson, M. Hank, D. Alsdorf, A. Ross, M. Clark, X. Liu, and J. Che, Bright spots, structure, magmatism in southern Tibet from INDEPTH seismic reflection profiling, Science, 274, 16881690,1996

Chen, W. P. and P. Molnar, Constraints on the seismic wave velocity structure beneath the Tibetan Plateau and their tectonic implications, J. Geophys. Res., 86, 5937-5962, 1981 .

Chun, K. Y. and T. Yoshii, Crustal structure of the Tibetan Plateau: A surface wave study by a moving window analysis, Bull. Seis. Soc. Am., 67, 1529-1540, 1977.

Clayton, R. and B. Engquist, Absorbing boundary conditions for acoustic and elastic wave propagations, Bull. Seis. Soc. Am., 67, 1529-1540, 1977.

Curtis, A. and J. H. Woodhouse, Crust and upper mantle shear velocity structure beneath the Tibetan plateau and surrounding regions from interevent surface phase velocity inversion, J. Geophys. Res., 102, 11,789-11,813, 1997.

Dahlen, F. A. and J. Tromp, Theoretical Global Seismology, pp. 449, Princeton Univ. Press, 1998.

Dziewonski, A. M. and A. L. Hales, Numerical analysis of dispersed seismic waves, in Method in Computational Physics, Volume 11, edited by B. A. Bolt, pp. 39-85, Academic Press, 1972.

Hirn, A., J. C. Lepine, G. Jobert, M. Sapin, G. Wittlinger, X. Z. Xin, G. E. Yuan, W. X. Jing, T. J. Wen, X. S. Bai, M. R. Pandefy, and J. J. Tater, Crustal structure and variability of the Himalayan border of Tibet, Nature, 307, 23-25, 1984.

Honda, S. and H. Sakai, Himalaya sanmyaku no keisei II, Kagaku, 58, 570-579, 1988 (in Japanese).

Its, E. N., Calculation of reflection and transmission coefficients, in Seismic Surface Waves in a Laterally Heterogeneous Earth, edited by V. I. Keilisborok, pp. 112-127, Kluwer Academic Publishers, The Netherlands, 1989.

Kennett, B. L. N., Guided wave propagation in laterally varying media-I. Theoretical development, Geophys. J. R. Astr. Soc., 79, 235-255, 1984.

Kind, R., J. Ni, W. Zhao, J. Wu, X. Yuan, L. Zhao, E. Sandivol, C. Reese, J. Nabelek, and T. Hearn, Evidence from earthquake data for a partially molten crustal layer in southern Tibet, Science, 274, 1692-1694, 1996.

Landisman, M., A. Dziewonski, and Y. Sato, Recent improvement in the analysis of surface wave observations, Geophys. J. R. Astr. Soc., 17, 369403, 1969.

Levander, A. L., Finite difference calcualtions of dispersive Rayleigh wave propagation, Tectonophys., 113, 1-30, 1985.

Levshin, A. L. and M. N. Ritzwoller, Characteritics of surface waves generated by events on and near the Chinese nuclear test site, Geophys. J. Int., 123, 131-148, 1995.

Levshin, A. L., L. Ratnikova, and J. Berger, Peculiarities of Surface-wave
Propagation across continental Eurasia, Bull. Seis. Soc. Am., 82, 24642493, 1992.

Makovsky, Y., S. L. Klempere, L. Ratschbacher, L. D. Brown, M. Li, W. $Z$ hao, and F. Meng, INDEPTH wide-angle reflection observation of $P$ wave-to- $S$-wave conversion from crustal bright spots in Tibet, Science, 274, 1690-1691, 1996.

Malishewsky, P., Surface Waves and Discontinuities, pp. 170-174, Elsevier, Amsterdam, 1987.

Meissner, R., The Continental Crust, A Geophysical Approach, International Geophysics Series, vol. 34, pp. 265, Academic Press, 1986.

Molnar, P., A review of geophysical constraints on the deep structure of the Tibetan Plateau, the Himalaya and the Karakoram, and their tectonic implications, Phyil. Trans. R. Soc. Lond., A, 326, 33-88, 1988.

Momoi, T., Scattering of Rayleigh waves by a semi-circular rough surface on layered media, Bull. Earthq. Res. Inst., Univ. Tokyo, 62, 163-200, 1987.

Nelson, K. D., W. Zhao, L. D. Brown, J. Kuo, J. Che, X. Liu, S. L. Klempere, Y. Makovsky, R. Meissner, J. Mechie, R. Kind, F. Wenzel, J. Ni, J. Nabelek, C. Leshou, H. Tan, W. Wei, A. G. Jones, J. Booker, M. Unsworth, W. S. Kidd, M. Hauck, D. Alsdorf, A. Ross, M. Cogan, C. Wu, E. Sandvol, and M. Edwards, Partially molten middle crust beneath southern Tibet: Synthesis of project INDEPTH results, Science, 274, 1684-1688, 1996.

Nolet, G., The upper mantle under Western Europe inferred from the dispersion of Rayleigh waves, J. Geophys., 43, 265-285, 1977.

Pedersen, H. A., V. Maupin, and M. Campillo, Wave diffraction in multilayered media with the indirect boundary element method: application to 3-D diffraction of long-period surface waves by 2-D lithospheric structures, Geophys. J. Int., 125, 545-558, 1996.

Pedersen, H. A., J. P. Avouac, and M. Campillo, Anomalous surface waves from Lop Nor nuclear explosions: Observations and numerical modelling, J. Geophys. Res., 103, 15,051-15,068, 1998.

Pollitz, F. F., Surface wave scattering from sharp lateral discontinuities, $J$. Geophys. Res., 99, 21,891-21,909, 1994.

Romanowicz, B. A., Constraints on the structure of the Tibet Plateau from pure path phase velocities of Love and Rayleigh waves, J. Geophys. Res. 87, 6865-6883, 1982.

Saito, M., DISPER80: A subroutine package for the calculation of seismic normal mode solutions, in Seismic Algorithms, pp. 293-319, Academic Press, 1988

Sato, Y., Dansei Hadouron (Theory of Elastic Wave Motions), pp. 252-253, Iwanami Shoten, 1978 (in Japanese).

Schwab, F. A. and L. Knopoff, Fast surface waves and free mode computations, in Methods in Computational Physics, Volume 11, edited by B. A Bolt, pp. 87-180, Academic Press, 1972.

Snieder, R., 3-D linearized scattering of surface waves and a formalism for surface wave holography, Geophys. J. R. Astr. Soc., 84, 581-605, 1986.

Snieder, R., Large-scale waveform inversions of surface waves for Lateral heterogeneity 2. Application to surface waves in Europe and the Mediterranean, J. Geophys. Res., 93, 12,067-12,080, 1988.

Tanimoto, T., Modelling curved surface wave path: membrane surface wave synthetics, Geophys. J. Int., 102, 89-100, 1990.

Tanimoto, T., Crustal structure of the Earth, Global earth physics, in $A$ Handbook of Physical Constants, pp. 214-224, edited by T. J. Ahrens, AGU, 1995.

Wittlinger, G., F. Masson, G. Poupinet, P. Tapponnier, J. Mei, G. Herquel, J. Guilbent, U. Achauer, X. Guanqi, S. Danian, and Lithoscope Kunlun Team, Seismic tomography of northern Tibet and Kunlun: Evidence for crustal blocks and mantle velocity constants, Earth Planet. Sci. Lett., 139, 263-279, 1996.

Wu, F. T. and A. L. Levshin, Surface-wave group velocity tomography of East Asia, Phys. Earth Planet. Int., 84, 59-77, 1994.

Wu, F. T., A. L. Levshin, and V. M. Kozhevnikov, Rayleigh wave group velocity tomography of Siberia, China and the vicinity, Pure Appl. Geophys., 149, 447-473, 1997.

Yamazaki, K. and H. Ishii, Study of phase and group velocities in a dipping layer overlying an elastic medium using theoretical sismograms, J. Phys. Earth, 21, 445-462, 1973.

Yoshida, M., Spectra of the first higher mode of simulated oceanic Rayleigh waves generated by deep earthquakes of the dip-slip type, Bull. Earthq. Res. Inst., Univ. Tokyo, 57, 609-625, 1982.

Yoshida, M., Study on the propagation of Love waves across irregular structures of the Moho discontinuity, Bull. Earthq. Res. Inst., Univ. Tokyo, 71 103-236, 1996.

M. Yoshida (e-mail: mitsuru@eri.u-tokyo.ac.jp) 